Article

\title{
Reliability Assessment of Distribution Networks with Optimal Coordination of Distributed Generation, Energy Storage and Demand Management
}

\author{
Alberto Escalera ${ }^{1,2}, *\left(\mathbb{1}\right.$, Edgardo D. Castronuovo ${ }^{2}\left({ }^{\infty}\right.$, Milan Prodanović $^{1}$ and \\ Javier Roldán-Pérez ${ }^{1}$ (D) \\ 1 IMDEA Energy Institute, 28935-Mostoles, Madrid, Spain \\ 2 Department of Electrical Engineering, University Carlos III de Madrid, Avda. Universidad 30, \\ 28911-Leganés, Madrid, Spain \\ * Correspondence: alberto.escalera.blasco@gmail.com
}

Received: 20 July 2019; Accepted: 13 August 2019; Published: 20 August 2019

check for updates

\begin{abstract}
Modern power distribution networks assume the connection of Distributed Generators (DGs) and energy storage systems as well as the application of advanced demand management techniques. After a network fault these technologies and techniques can contribute individually to the supply restoration of the interrupted areas and help improve the network reliability. However, the optimal coordination of control actions between these resources will lead to their most efficient use, maximizing the network reliability improvement. Until now, the effect of such networks with optimal coordination has not been considered in reliability studies. In this paper, DGs, energy storage and demand management techniques are jointly modelled and evaluated for reliability assessment. A novel methodology is proposed for the calculation of the reliability indices. It evaluates the optimal coordination of energy storage and demand management in order to reduce the energy-not-supplied during outages. The formulation proposed for the calculation of the reliability indices (including the modelling of optimal coordination) is described in detail. The methodology is applied to two distribution systems combining DGs, energy storage and demand management. Results demonstrate the capability of the proposed method to assess the reliability of such type of networks and emphasise the impact of the optimal coordination on reliability.
\end{abstract}

Keywords: distribution networks; energy storage; demand management; reliability assessment; optimal restoration

\section{Introduction}

Reliability is a fundamental parameter in the design of power systems as it determines the quality of service offered to customers [1,2]. This service itself is adversely affected by network component failures causing supply interruptions and different actions are commonly applied to mitigate the negative effects of network failures [3,4]. In recent years new generation and storage technologies as well as demand response techniques have been widely deployed in power distribution networks. In addition to their primary role they can be also used to improve the reliability of the system [5]. Among these technologies, the application of Distributed Generators (DGs) represents an effective solution for supplying power to the areas interrupted by network faults. The intentional islanding operation is a mechanism that can be used to improve reliability in isolated areas that would be otherwise disconnected [6,7]. However, a significant part of DGs use renewable energy resources like wind or solar and, consequently, are exposed to seasonal variability and intra-day fluctuations that limit their capacity to restore the interrupted supply and improve reliability [8]. Under these circumstances, 
energy storage devices and demand management can be used to compensate fluctuations of renewable generation and minimise the impact of interruptions [9]. Therefore, the flexibility introduced by these energy balancing resources needs to be considered during the calculation of reliability indices.

Reliability of power distribution networks is typically evaluated by using probabilistic techniques either based on analytical or simulation approaches [10]. In recent years, these techniques have been extended to assess renewable generation and possible islanded network operation [11-15]. In these works, variability of renewable resources is evaluated during outages as it can be insufficient to restore all the demand in isolated areas. Under these circumstances, the effect of applying demand response actions to support renewable generation has been studied in References [16-18]. Another option to improve reliability is energy storage, studied in References [19-21] for energy storage devices and in References [22,23] for parking lots. In these works, demand response and energy storage options are individually used to support renewable generation. If both options are combined, further reliability improvement can be obtained, as analysed in this paper.

The combined operation of energy storage and demand response offers additional flexibility in the supply restoration. Different real-time restoration strategies can be used to manage energy storage and demand response during an interruption, producing different impacts on reliability. Therefore, the choice of the restoration strategy potentially has a significant impact on the reliability evaluation. Generally, these strategies aim to minimise the impact of interruptions but they are typically too complex to be addressed by using probabilistic reliability studies [24]. Moreover, their evaluation in reliability studies requires from increased computation efforts, especially when a large number of faults and conditions are assessed [25]. To reduce the mentioned complexity and computational burden, simple restoration strategies are commonly considered in reliability studies [25]. Yet, the impact of more complex restoration strategies, including in some cases the application of optimisation techniques, has been evaluated in specific studies for realistic evaluation. For example, the contribution of optimal switching operation is considered in Reference [24], optimal load shedding is evaluated in References [18,26], optimal operation of parking lots together with renewable DGs is assessed in Reference [22], and optimal management of multiple microgrids supporting active distribution networks in Reference [27]. However, none of the mentioned references takes into account optimal and coordinated management of DGs, energy storage and dispatchable loads in the calculation of reliability, maybe because of the additional complexity in the formulation.

In this paper, a methodology is proposed to assess the reliability of distribution systems that jointly combine DGs, energy storage and demand management. The assessment is performed by including the optimal coordination of the mentioned resources (DGs, energy storage and demand management) during outages, operated in such way that the network reliability is improved. The methodology proposed for this assessment identifies the areas isolated by the faults and models the uncertainties of generation and demand. Optimal control actions of energy storage and load shedding are calculated for each generation and demand condition and included in the calculation of reliability indices. The main contributions of this paper are:

1. The effects of DGs, energy storage and dispatchable loads are jointly modelled in the proposed methodology for reliability assessment. The resources are comprehensively represented in a unique model, including:

- Renewable and conventional DGs.

- Uncertainties of generation and demand, because their chronological fluctuations.

- Dispatchable and non-dispatchable loads. Dispatchable loads are formed of several portions or levels of load shedding.

- Priorities in the restoration of loads or portions of loads.

- Chronological relationship and uncertainties in the level of state-of-charge (SOC) of the storage. 
2. Optimisation is applied to model the coordination of the resources with an aim to improve the reliability of the system. The following formulations are proposed for this purpose:

- A mixed integer linear optimisation problem is proposed, in order to obtain the optimal solution and reduce the computation burden. Minimisation of the energy-not-supplied to critical customers during faults is sought.

- An analytical approach is used for efficient evaluation of the reliability indices. As the literature shows, the analytical approach demands less computation resources than the alternative Monte Carlo simulation [25]. However, note that the optimisation problem can be easily adapted to Monte Carlo simulation, if necessary.

- Monthly and hourly variations of demand and generation are modelled in compliance with the analytical formulation. Their chronological dependencies are properly modelled for the accurate evaluation of the the optimal coordination.

3. Additional operational alternatives not yet considered by existing reliability assessment methodologies are modelled and studied, including: more than one interruption during a fault, coordination of non-dispatchable and dispatchable loads with demand-control levels, and possible hourly constraints for energy storage operation. The effect of these criteria in the reliability calculation is evaluated.

4. Comparative and sensitivity studies can be readily performed by using the proposed algorithm and applied to calculate the consequences of the resources and parameters modelled in a very complex reliability assessment. The impact of optimal coordinated restoration, energy storage size, number and levels of dispatchable loads, and more than one interruption per fault, among others, is evaluated and critically analysed in this work.

The obtained results are used to demonstrate that DGs, energy storage and demand management have an important and beneficial impact on the reliability of distribution networks. However, in order to obtain significant additional improvements, the modelled resources must be jointly integrated and operated in an optimal and coordinated way.

The paper is organised as follows: the problem and the overview of the proposed solution are defined in Section 2. Section 3 introduces the procedure to calculate the reliability indices, while Section 4 presents the model for the generation and demand uncertainties. In Section 5, the procedure to assess the contribution of coordinated restoration to reliability is described, including the details of the optimal restoration. In Section 6 the case study is presented. Finally, the conclusions are discussed in Section 7.

\section{Problem Definition and Solution Proposed}

\subsection{Problem Definition}

Figure 1 shows the single-line diagram of a radial distribution network and the isolated area created when the fault $j$ is cleared. This area incorporates DGs (conventional and renewable) and energy storage devices that are used to supply power during outages. This area also includes non-dispatchable and dispatchable loads. The dispatchable loads are formed of different levels (or steps of load) that can be independently controlled just as it is shown in Figure 1 for bus 17. It is assumed that the isolated area is equipped with the protection devices and control systems required to operate isolated from the main grid. More information of these technologies can be found in Reference [28].

Figure 2 shows the demand and generation profiles of the isolated area in Figure 1. The time required to isolate and configure the isolated area is called $s w_{j}$ (switching time), while the time required to fix a defective element is called $r_{j}$ (repair time). Supply restoration is evaluated along the repair time. As shown in Figure 2, the power generated by the DGs is insufficient to supply the demand between the time instants $i t_{2}$ and $i t_{3}$. During this time interval energy storage devices and load shedding strategies allow the selective load restoration and reduce the impact of the interruption. 


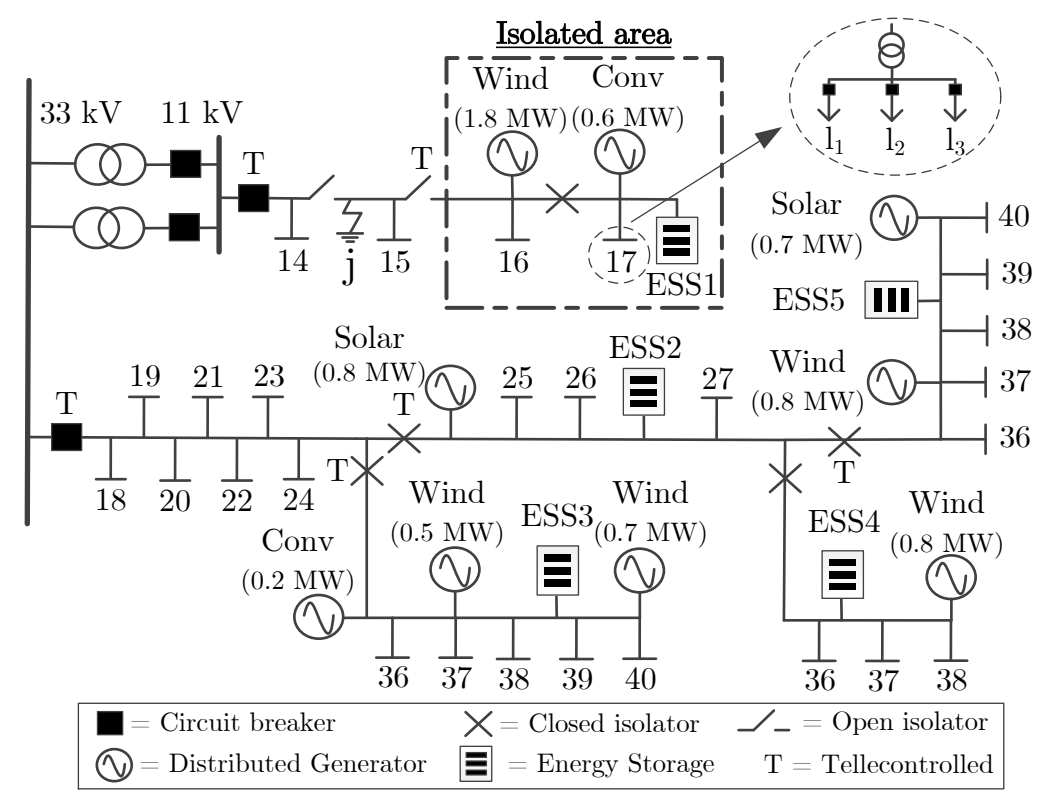

Figure 1. Bus 6 Roy Billinton Test System [29], with an isolated area equipped to be operated in islanded mode.

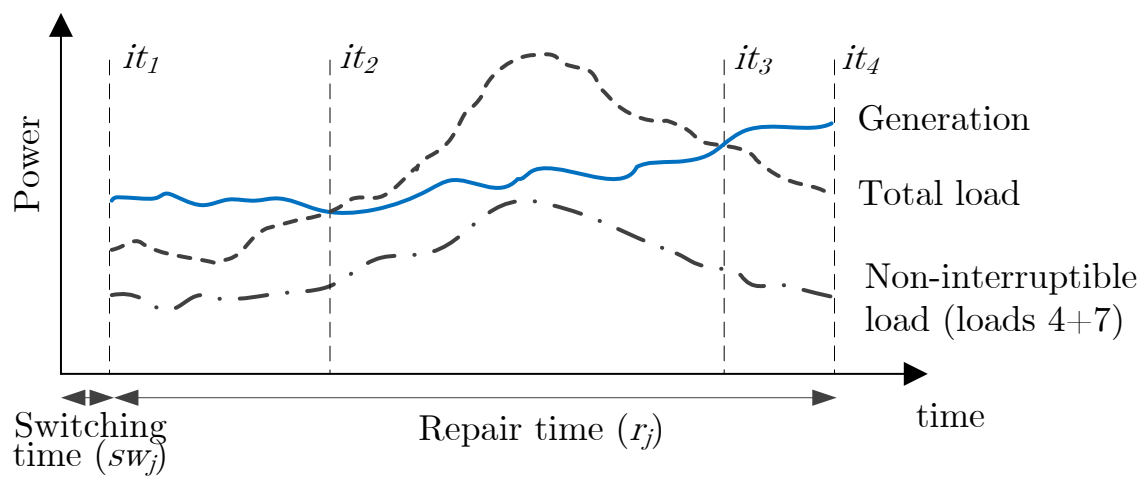

Figure 2. Example of generation and load during the fault of the isolated area in Figure 1.

Energy storage and load shedding introduce flexibility to supply restoration. This means they can be operated in different ways over the fault duration and a coordinated strategy that minimises the impact of interruptions can be used [30]. The application of the optimal strategy has an effect on the reliability indices of the customers within the isolated areas and this effect should be included in the reliability assessment of active distribution networks.

\subsection{Overview of the Proposed Method}

Figure 3 shows the proposed method for the reliability assessment of active distribution networks. It includes the new calculation steps proposed to evaluate the contribution of optimally-restored isolated areas (marked between dashed lines in the figure). Other features of the method are: (1) it is a generalised method that can also incorporate other restoration strategies, (2) it uses the analytical approach (yet it could be readily extended to Monte Carlo simulation based techniques), (3) it uses the zone branch methodology proposed in Reference [31] to address fault isolation and restoration actions, (4) it neglects failures of the protection devices (assumption commonly accepted in reliability studies [10]), and (5) average values of failure statistics are used for network components (an usual input data for analytical techniques [10]). 


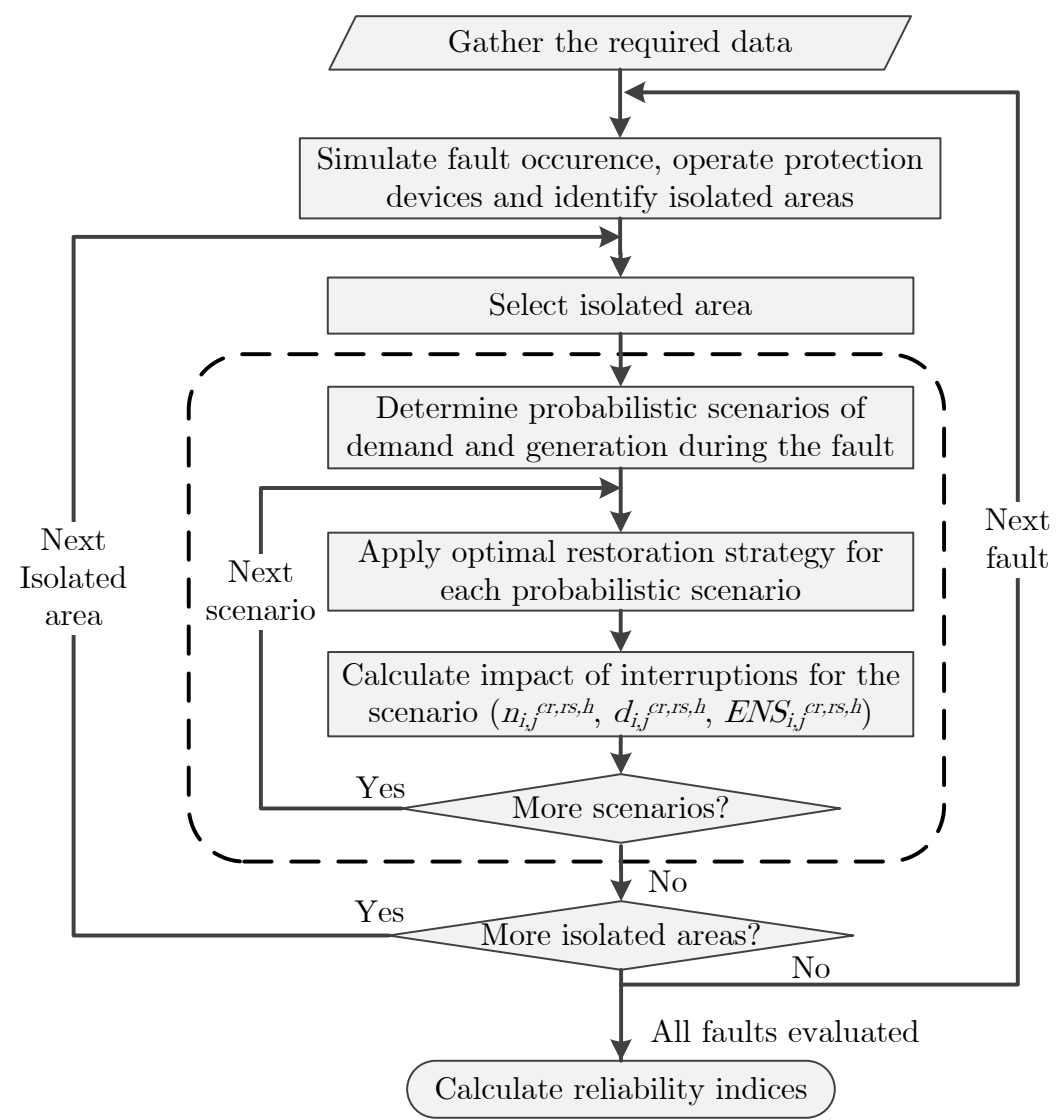

Figure 3. Proposed method for the reliability assessment of active distribution networks with optimal restoration of isolated areas.

The first step to assess reliability is to gather all the required data (it is assumed that data are robust and reliable). Then, the impact of each component failure is evaluated, and this starts by simulating the operation of the protection devices and by identifying the isolated areas created [31]. For each isolated area, probabilistic scenarios for demand and generation are defined over a year (more details in Section 4). After that, the adequacy of generation and demand is assessed for each defined scenario. The procedure described in Section 5 is used to model the optimal restoration and to determine the impact of the interruptions. Finally, the reliability indices are calculated.

\section{Reliability Indices Calculation}

Area reliability indices defined in Reference [32] are used to evaluate the reliability of a distribution system. Among these indices, SAIFI (System Average Interruption Frequency Index), SAIDI (System Average Interruption Duration Index) and ENS (Energy Not Supplied) are commonly used, and they are defined as [32]:

$$
\begin{gathered}
\text { SAIFI }=\frac{\sum_{i=1}^{N_{i}} N c_{i} \lambda_{i}}{\sum_{i=1}^{N_{i}} N c_{i}}, \\
\text { SAIDI }=\frac{\sum_{i=1}^{N_{i}} N c_{i} U_{i}}{\sum_{i=1}^{N_{i}} N c_{i}}, \\
\text { ENS }=\sum_{i=1}^{N_{i}} E N S_{i},
\end{gathered}
$$

where $i$ and $N_{i}$ design the index and number of load points in the network, and $N c_{i}$ the number of customers in load point $i$. 
The reliability indices of a load point (failure rate $\lambda_{i}$, outage duration $r_{i}$ and annual unavailability $\left.U_{i}\right)$, and the related energy-not-supplied $E N S_{i}$ are calculated as [32]:

$$
\begin{aligned}
\lambda_{i}=\sum_{j=1}^{N_{j}} \lambda_{i, j}, \quad U_{i} & =\sum_{j=1}^{N_{j}} \lambda_{j} r_{i, j}, \quad r_{i}=\frac{U_{i}}{\lambda_{i}}, \\
E N S_{i} & =\sum_{j=1}^{N_{j}} E N S_{i, j},
\end{aligned}
$$

where $j$ and $N_{j}$ are the index and number of evaluated failures and $\lambda_{j}$ is the annual rate of failure $j$. In addition, variables $\lambda_{i, j}, r_{i, j}$ and $E N S_{i, j}$ are the failure rate, interruption duration and energy-not-supplied of load point $i$ caused by failure $j$, and their calculation depends on whether the load point is restored or not by islanded operation during the failure.

\subsection{Load Points without Islanded Restoration}

The commonly-used procedure in Reference [10] is applied to calculate $\lambda_{i, j}, r_{i, j}$ and $E N S_{i, j}$. It depends on the segment where the load point is located and the presence of alternatives to restore the supply.

\subsection{Load Points with Islanded Restoration}

In this case $\lambda_{i, j}, r_{i, j}$ and $E N S_{i, j}$ are calculated by using the formulation proposed in this paper:

$$
\begin{aligned}
& \lambda_{i, j}=\lambda_{j} \sum_{s n}^{N_{s n}} p_{s n} n_{i, j}^{s n}, \\
& r_{i, j}=\sum_{s n}^{N_{s n}} p_{s n} r_{i, j}^{s n} \\
& E N S_{i, j}=\lambda_{j} \sum_{s n}^{N_{s n}} p_{s n} E N S_{i, j}^{s n}
\end{aligned}
$$

where $s n$ denotes the scenario of generation and demand, $N_{s n}$ the number of evaluated scenarios, and $p_{s n}$ the probability of the scenario. These scenarios of generation and demand are defined and calculated by using the modelling technique proposed in Section 4 . With regard to variables $n_{i, j}^{s n}, r_{i, j}^{s n}$ and $E N S_{i, j}^{s,}$, they represent the number of interruptions, their duration and the energy-not-supplied for scenario sn. These variables are calculated as described in Section 5, where a novel formulation that considers optimal coordination in order to calculate reliability indices is proposed.

\section{Renewable Generation and Demand Modelling}

Renewable generation and demand uncertainties are modelled by using specific probabilistic scenarios. These scenarios represent the variability and time-dependency of generation and demand during outages. In addition, they include the state of charge (SOC) uncertainty of energy storage systems when a fault occurs. Such modelling allows the uncertainties to be assessed by using the analytical methodology.

\subsection{Generation and Demand Profiles During Outages}

The proposed scenarios use profiles as those shown in Figure 2 to model the time-dependency of renewable generation and demand during outages. These profiles are divided in time-steps in order to facilitate their evaluation. Each time-step has its own powers and duration in hours or fractions of hours.

Switching and repair times in Figure 2 are analysed separately because the restoration of supply is evaluated only during the repair time. For fault $j$, ts and $T s_{j}$ are defined as the index and set of 
time-steps in the switching time, while $t$ and $T_{j}$ are the index and set of time-steps in the repair time. Also $\delta t_{j}$ and $\delta t s_{j}$ are defined as the duration in hours of each time-step in the switching and repair times, respectively.

\subsection{Scenarios Calculation}

The following steps are performed to generate the scenarios of renewable generation, demand and SOC:

1. The period of one year is divided into representative time-intervals, for example, one day per month. Typical profiles of renewable generation and demand are obtained for these time-intervals as in Reference [21]. Moreover, typical profiles of SOC are included assuming normal operating conditions (they can be obtained from real data or by using algorithms [33]).

2. Different profiles of generation and demand are obtained during an outage. These profiles are taken from the typical profiles defined in (1) by assuming that the analysed fault may occur at any time-step of the representative time-interval. These profiles are assigned to the load points and renewable DGs in an isolated area. Moreover, the SOC in that time-step is assigned to each storage device representing its values when the fault occurs.

3. Probabilistic states of the DGs and storage devices in the isolated area are created as in Reference [21]. Each device has two possible states, up and down, that are combined for all the devices in the area resulting in a set of probabilistic states.

4. Generation profiles in (2) are combined with the devices states in (3) to obtain the generation-demand scenarios used in the reliability evaluation. Their annual probability $p_{s n}$ is determined as:

$$
p_{s n}=p_{g s} p_{r s} p_{h, r s} \quad \forall g s \in N_{g s}, r s \in N_{r s}, h \in N_{h, r s}
$$

where $p_{g s}, p_{r s}$ and $p_{h, r s}$ are the probabilities of generation state $g s$, representative time-interval $r s$ and time-step $h$ of time-interval $r s$, while $N_{g s}, N_{r s}$ and $N_{h, r s}$ are the numbers of generation states, time-intervals and time-steps per interval.

\section{Modelling Optimal Restoration in Reliability Assessment}

\subsection{Impact of the Interruptions}

This Section describes the proposed procedure to calculate the number of interruptions $n_{i, j}^{s n}$, their duration $r_{i, j}^{s n}$ and energy not supplied $E N S_{i, j}^{s n}$ (variables defined in Section 3). These variables measure the impact of a fault on customers within a post-fault isolated area and include the effect of the optimal and coordinated restoration using DGs, energy storage, dispatchable and non-dispatchable loads. In the rest of the Section the superscript $s n$ is not used in order to simplify the notation.

The calculation of $n_{i, j}, r_{i, j}$ and $E N S_{i, j}$ differentiates between dispatchable and non-dispatchable load points. The dispatchable ones are equipped with several levels of load shedding as remedial action to decrease the demand during outages.

\subsubsection{Non-Dispatchable Load Points}

In the isolated area, the impact of a fault on the load points that are not equipped with load shedding capability (index $m$, set $S_{m}$ ) is calculated as:

$$
n_{i, j}=\sum_{t=1}^{T_{j}} R n^{+}(t) \quad i \in S_{m}
$$




$$
\begin{aligned}
& r_{i, j}=s w_{j}+\sum_{t=1}^{T_{j}}(1-R n(t)) \delta t_{j}(t) \quad i \in S_{m} \\
& E N S_{i, j}=\sum_{t s=1}^{T s_{j}} P b_{m}(t s) \delta t s_{j}(t s)+\sum_{t=1}^{T_{j}} P b_{m}(t)(1-R n(t)) \delta t_{j}(t) \quad i \in S_{m}
\end{aligned}
$$

where $P b_{m}$ is the parameter representing the demand profile during the fault, $R n(t)$ is a binary variable equal to 1 if all the non-dispatchable loads are restored at time-step $t$, and $R n^{+}(t)$ is a binary variable equal to 1 only if all the non-dispatchable loads change from non-supplied to supplied state between $t-1$ and $t$. The other parameters related to switching time $\left(t s_{j}, T s_{j}, \delta t s_{j}\right)$ and repair time $\left(t_{j}, T_{j}, \delta t_{j}\right)$ are as previously defined. Variables $R n$ and $R n^{+}$are two unique variables used to represent all the non-dispatchable load points in the isolated area because these loads have to be restored together at the same time-step. In (10), the number of supply interruptions during the fault is stored $\left(R n^{+}(t)=1\right.$ represents an interruption). In (11), the interruption time includes the switching time and the non-restored time-steps during the repair time $\left(R_{n}(t)=1\right.$ means that the load is supplied in $\left.t\right)$. The energy-not-supplied is calculated as shown in (12), and includes the energy-not-supplied during the switching time (first summation) and during the repair time (second summation).

\subsubsection{Dispatchable Load Points}

Dispatchable load points (index $k$, set $S_{k}$ ) are equipped with several levels of load shedding (index $l$, set $S_{k, l}$ ) as defined in Section 2.1. The number of interruptions experienced by the customers in each level $\left(n_{i, j, l}\right)$ is given by:

$$
n_{i, j, l}=\sum_{t=1}^{T_{j}} R s_{k, l}^{+}(t) \quad i \in S_{k}, l \in S_{k, l}
$$

where $R s_{k, l}^{+}(t)$ is a binary variable equal to 1 if the level $l$ changes from non-supplied to supplied state between $t-1$ and $t$ (otherwise the variable is equal to 0 ).

The interruption duration caused by the fault within load shedding level $l$, designed as $r_{i, j, l}$, is:

$$
r_{i, j, l}=s w_{j}+\sum_{t=1}^{T_{j}}\left(1-R s_{k, l}(t)\right) \delta t_{j}(t) \quad i \in S_{k}, l \in S_{k, l}
$$

where $R s_{k, l}(t)$ is a binary variable equal to 1 if level $l$ is restored at time-step $t$ (otherwise it is 0 ).

Finally, the energy-not-supplied over the fault duration is defined for each level $l$ as $E N S_{i, j, l}$ and calculated as:

$$
E N S_{i, j, l}=\sum_{t s=1}^{T s_{j}} P b_{k, l}(t s) \delta t s_{j}(t s)+\sum_{t=1}^{T_{j}} P b_{k, l}(t)\left(1-R_{k, l}(t)\right) \delta t_{j}(t) \quad i \in S_{k}, l \in S_{k, l}
$$

where $P b_{k, l}$ is a parameter representing the discretized demand profile over the fault for level $l$ of load point $k$.

Then, the overall impact of the fault on a load point $k$ is calculated by aggregating the impacts of all its levels:

$$
\begin{array}{cc}
n_{i, j}=\frac{\sum_{l \in S_{k, l}} n_{i, j, l} N c_{i, l}}{N c_{i}} & i \in S_{k}, \\
r_{i, j}=\frac{\sum_{l \in S_{k, l}} r_{i, j, l} N c_{i, l}}{N c_{i}} & i \in S_{k}, \\
E N S_{i, j}=\sum_{l \in S_{k, l}} E N S_{i, j, l} & i \in S_{k},
\end{array}
$$


where $N c_{i, l}$ is the number of customers for level $l$.

The values of the binary variables $R n, R n^{+}, R s_{k, l}$ and $R s_{k, l}^{+}$depend on the strategy applied to restore the supply. Multiple options for energy storage operation and multiple dispatchable loads, each one with several levels of load shedding, lead to numerous possible solutions of these binary variables and, therefore, multiple restoration options. Instead of adopting heuristic or approximate approaches to determine the solution of this complex systems, a mixed integer linear problem is defined in Sections 5.2 and 5.3. Such problem formulation allows to evaluate the impact of the optimal restoration on reliability indices.

\subsection{Equality and Inequality Constraints}

\subsubsection{Adequacy Assessment}

The active power adequacy in the isolated area is a fundamental criterium for reliability assessment of distribution networks with islanded operation [11-14,16,18]. Here it is evaluated for each time-step of the fault by using:

$$
R n(t) \sum_{m \in S_{m}} P b_{m}(t)+\sum_{k \in S_{k}} \sum_{l \in S_{k, l}} R s_{k, l}(t) P b_{k, l}(t)+\sum_{s \in S_{s}} P c_{s}(t) \leq \sum_{g \in S_{g}} P g_{g}(t)+\sum_{s \in S_{s}} P d_{s}(t) \quad \forall t
$$

where $g$ and $S_{g}$ are the index and set of DGs in the isolated area, $s$ and $S_{s}$ are the index and set of energy storage systems, $P g_{g}$ is a parameter representing the power profile that DG $g$ can generate over the fault duration, while $P c_{s}$ and $P d_{s}$ are continuous variables of the power charging and discharging the energy storage $s$.

The inequality in (19) guarantees that generation-demand adequacy is preserved, then no additional variables are needed for modelling the power balance in the area. Reactive power balance and network constraints are not included in the evaluation as they are typically considered in other stages of the network planning. This is a common assumption used for reliability assessment of active distribution networks $[11-14,16,18]$ that also helps to keep the linearity of the optimisation problem.

\subsubsection{Energy Storage}

The chronological charge and discharge of the energy storage systems (ESS) are modelled by:

$$
\begin{array}{lr}
S O C_{s}(t+1)=S O C_{s}(t)+\frac{\delta t_{j}(t)}{C_{s}}\left(P c_{s}(t) \eta c_{s}-\frac{P d_{s}(t)}{\eta d_{s}}\right) & \forall s \in S_{s}, \forall t \\
\left(\underline{P c_{s}}, \underline{P d}_{s}\right) \leq\left(P c_{s}(t), P d_{s}(t)\right) \leq\left(\overline{P c}_{s}, \overline{P d}_{s}\right) & \forall s \in S_{s}, \forall t \\
\underline{S O C}_{s} \leq S O C_{s}(t) \leq \overline{S O C}_{s} & \forall s \in S_{s}, \forall t \\
S O C_{s}(1)=\operatorname{SOCini}_{s} & \forall s \in S_{s}
\end{array}
$$

where $S O C_{S}$ is a continuous variable with the evolution of the SOC over the fault duration, SOCini is the initial SOC when the fault occurs (given in the scenarios defined in Section 4 ), $C_{S}$ is the storage capacity, $\eta c_{S}$ and $\eta d_{s}$ are the efficiencies to charge and discharge, parameters $S O C_{S}, \underline{P c_{S}}$ and $\underline{P d_{S}}$ indicates the minimum limit of the associated variables, while $\overline{S O C}_{s}, \overline{P C}_{s}$ and $\overline{P d}_{S}$ the maximum limit.

In addition, the difference in the stored energy between the start and the end of the fault is defined as $\delta D_{S}$ and calculated as in (24). It assumes the final SOC $\left(S_{S O C}(T+1)\right)$ does not exceed the initial SOC and, in this way, unnecessary charges are avoided during the outage period.

$$
\delta D_{s}=\frac{\operatorname{SOCini}_{s}-\operatorname{SOC}_{s}(T+1)}{\delta t_{j}(t)}, \delta D_{s} \geq 0 \quad \forall s \in S_{s}
$$




\subsubsection{Load Shedding}

According to the topology of the network isolated areas described in Section 2, the dispatchable loads are restored only if the non-dispatchable loads are also restored and there is still additional energy to be supplied. The binary variables with the restoration state $\left(R n\right.$ and $\left.R s_{k, l}\right)$ are used for its modelling:

$$
R s_{k, l}(t) \leq R n(t) \quad \forall t, \forall k, \forall l \in S_{k, l}
$$

\subsubsection{Number of Interruptions during a Fault}

Additional constraints are required to limit the number of interruptions and restorations happened during a fault to customers. This is an important parameter when fluctuations of renewable generation and demand are considered. One option is to set the number of interruptions to one [30]. Yet, permitting additional interruptions can reduce the outage duration and the energy-not-supplied. Consequently, a more extended formulation is proposed here for a generic number of permitted interruptions $L I$. The formulation for the non-dispatchable loads is:

$$
\begin{array}{ll}
\sum_{t=1}^{T_{j}+1} R n^{+}(t) \leq L I & \\
R n(t)-R n(t-1)=R n^{+}(t)-R n^{-}(t) & t=1, . ., T_{j}+1 \\
R n^{+}(t)+R n^{-}(t) \leq 1 & t=1, . ., T_{j}+1 \\
R n^{+}, R n^{-} \in\{0,1\} &
\end{array}
$$

where the binary variable $R n^{-}$takes the value of 1 if supply changes from supplied to not-supplied states, while $R n^{+}$the value of 1 if load changes from not-supplied to supplied state as previously defined.

Constraint (26) guarantees that the number of transitions from interrupted to supplied states is lower than the limit LI; constraint (27) determines the transitions from not-supplied to supplied states and viceversa; and (28) avoids simultaneous transitions in variables $R n^{+}(t)$ and $R n^{-}(t)$. These equations preserve the linearity of the optimisation problem.

In the case of dispatchable loads, the number of interruptions is limited by extending Equations (26)-(29) to each level of load shedding as follows:

$$
\begin{aligned}
& \sum_{t=1}^{T_{j}+1} R s_{k, l}^{+}(t) \leq L I \quad \forall k, \forall l \in S_{k, l} \\
& R s_{k, l}(t)-R s_{k, l}(t-1)=R s_{k, l}^{+} t-R s_{k, l}^{-}(t) \quad \forall k, \forall l \in S_{k, l}, t=1, . ., T_{j}+1 \\
& R s_{k, l}^{+}(t)+R s_{k, l}^{-}(t) \leq 1 \quad \forall k, \forall l \in S_{k, l}, t=1, . ., T_{j}+1 \\
& R s_{k, l}^{+}, R s_{k, l}^{-} \in\{0,1\} \quad \forall k, \forall l \in S_{k, l}
\end{aligned}
$$

where the binary variable $R s_{k, l}^{-}$measures the transitions from supplied to not-supplied states for each level of load shedding, and $R s_{k, l}^{+}$the transitions from not-supplied to supplied states as previously defined.

\subsection{Optimisation Problem}

The optimisation problem with objective function (34) and constraints (19)-(33) is proposed to calculate the continuous variables $P c_{s}, P d_{s}, S O C_{s}$ and $\delta D_{s}$, and the binary variables $R n, R n^{+}, R s_{k, l}$ 
and $R s_{k, l}^{+}$. These binary variables are used in (10)-(15) to calculate the reliability indices, thus the optimal coordination is considered in the calculation.

$$
\operatorname{Max} \sum_{t=1}^{T_{j}}\left(\omega n R n(t) \sum_{m \in S_{m}} P b_{m}(t)+\sum_{k \in S_{k}} \sum_{l \in S_{k, l}} \omega_{k, l} R s_{k, l}(t) P b_{k, l}(t)-c_{1} \sum_{s \in S_{s}} P d_{s}(t)\right)+c_{2} \sum_{s \in S_{s}} \delta D_{s}
$$

The first two terms in (34) represent the restored energy in non-dispatchable and dispatchable loads. The restored energy is maximized (or the ENS reduced) for the considered contingency taking into account the priority of the loads. It means that the priority of the customers ( $\omega n$ for non-dispatchable and $\omega_{k, l}$ for dispatchable loads) is considered.

The third term avoids unnecessary energy storage discharges that do not increase the amount of restored energy, reducing in that way power releases. The fourth term aims to have a stored energy at the end of the fault as close as possible to the stored energy at the start of the fault. In the third and fourth terms, weighting factors $c_{1}$ and $c_{2}$ of small values are used (less than a tenth of the first two terms of (34)). These values are chosen to avoid any alteration of the optimal restoration results.

Optimization problem (19)-(34) is solved in GAMS, using CPLEX solver.

\section{Case Study}

\subsection{Test Network}

The proposed method is applied to study the reliability of two distribution systems with DGs, energy storage and dispatchable loads and considers the contribution of the optimal and coordinated restoration to reliability. The first system (shown in Figure 1) is formed by feeders 3 and 4 of Bus 6 Roy Billinton Test System [34], a well-know system for testing reliability assessment techniques that consider islanded operation [16,18]. The second system (shown in Figure 4) corresponds to a real $11 \mathrm{kV}$ radial feeder, larger than the first system and designed as Feeder c72. In these networks, islanded operation was evaluated for all the isolated areas caused by faults once reconfiguration actions are applied. In Figure 4, the dotted lines represent the islands created by the operator when faults occur in upstream segments of these dotted areas.

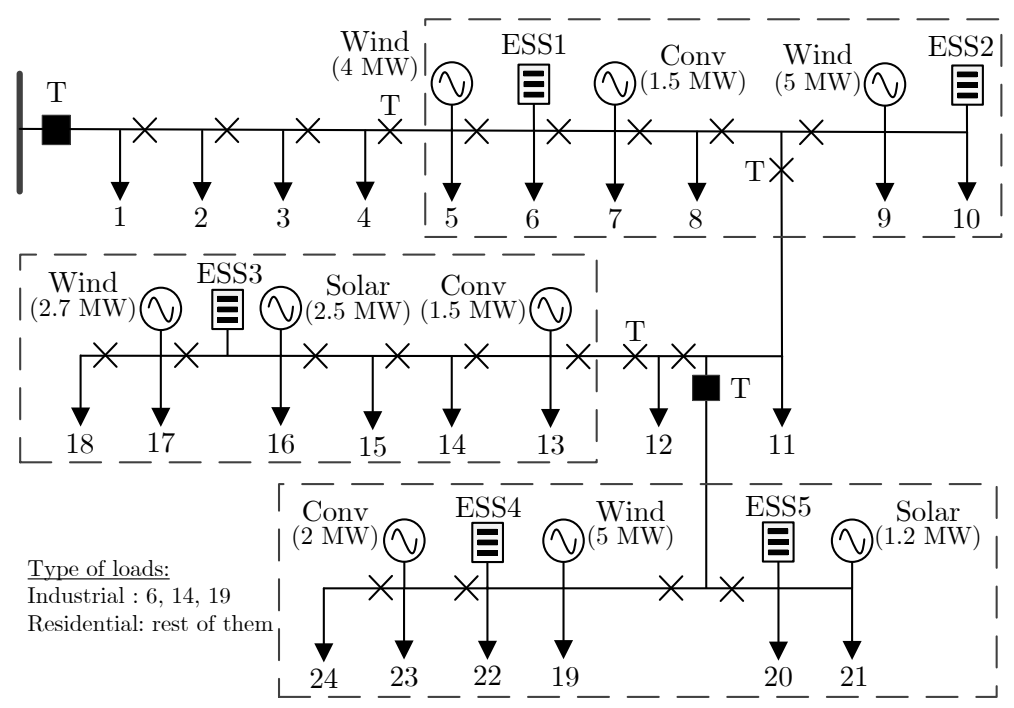

Figure 4. Single-line diagram of the second test system under study (Feeder c72).

The reliability indices in Reference [29] are used for lines and transformers. The switching time is $1 \mathrm{~h}$ for manual and $10 \mathrm{~min}$ for telecontrolled switches. The annual unavailability of the conventional, wind and solar generation is $0.006,0.028$ and 0.021 and their starting times $0.25 \mathrm{~h}$. The unavailability of energy storage is 0.004 . 
The data of demand, wind and solar powers in Reference [35] are used to create the scenarios. The procedure described in Section 4 is applied to consider the uncertainty of demand and renewable generation. The number of interruptions during a fault was limited to one $(L I=1)$ except for the analysis in Section 6.5.

Four cases are analysed to determine the impact of the optimal and coordinated restoration strategy on reliability:

1. DG only: DGs in Figures 1 and 4 were the unique resources used to restore the supply in the islands.

2. DG and Energy Storage (DG+ESS): the energy storage devices in Figures 1 and 4 were integrated into previous case, DG only. Their nominal capacities and powers are given in Table 1 . In addition, all the energy storage devices have: $\underline{P_{c}}$ and $\underline{P_{d}}$ of $0 \mathrm{MW}, \overline{S O C}$ of $0.9, \underline{S O C}$ of 0.1 and $\eta c, \eta d$ of 0.9 .

Table 1. Nominal capacity and rated powers of the energy storage.

\begin{tabular}{ccccccc}
\hline System & Parameter & ESS1 & ESS2 & ESS3 & ES4 & ESS5 \\
\hline \multirow{2}{*}{ Bus 6 } & $\mathrm{C}(\mathrm{MWh})$ & 1.75 & 0.8 & 1.2 & 0.75 & 1.5 \\
& $\overline{P_{c}}, \overline{P_{d}}(\mathrm{MW})$ & 0.35 & 0.16 & 0.24 & 0.15 & 0.3 \\
\hline Feeder & $\mathrm{C}(\mathrm{MWh})$ & 4 & 5 & 5.2 & 5 & 1.2 \\
c72 & $\overline{P_{c}}, \overline{P_{d}}(\mathrm{MW})$ & 0.8 & 1 & 1.04 & 1 & 0.24 \\
\hline
\end{tabular}

3. DG and Load Shedding (DG+LS): the load shedding functionality was added to the case DG only. Except in Section 6.4, all the results were obtained for a scenario with low level of dispatchable loads, and for one level of load shedding in these loads. The priority factors per customer type ( $\omega n$ and $\omega_{k, l}$ ) were 10 for commercial/industrial, 1.9 for small/residential and 0.5 for farms. The types of customers are specified in Reference [34] and in Figure 4.

4. DG, Energy Storage and Load Shedding (DG+ESS+LS): the three resources were coordinated and optimally operated for the selective restoration of the isolated areas.

The method proposed in this paper is applied to calculate the reliability in the four cases described above. As the same method is used for all the cases, the comparison among them is direct and realistic. Also, the effect of optimal coordination among DGs, energy storage and dispatchable loads is evaluated (this is the main objective of this case study). Since there are no other methods available in the literature to assess the reliability of distribution networks with optimally coordinated resources and the alternatives and models here considered, the proposed technique cannot be compared with other ones.

\subsection{Test Comparison}

The reliability indices of the test networks are compared for the four cases evaluated. Results of SAIDI and ENS are shown in Table 2, while the variations of these indices (referred to the case with only DG) are shown in brackets. The network SAIFI is the same for the four cases as the interruptions are not avoided but only reduced on duration and amount of energy-not-supplied.

Table 2. SAIDI (hours/customer year) and ENS (MWh/year) for the evaluated cases.

\begin{tabular}{cccccc}
\hline System & Index & DG & DG+ESS & DG+LS & DG+ESS+LS \\
\hline \multirow{2}{*}{ Bus 6 } & SAIDI & 8.6 & $8.3(-4.0 \%)$ & $7.9(-8.5 \%)$ & $6.7(-22.1 \%)$ \\
& ENS & 45.6 & $44.4(-2.8 \%)$ & $43.5(-4.7 \%)$ & $39.8(-12.7 \%)$ \\
\hline Feeder & SAIDI & 22.6 & $22.6(-0.3 \%)$ & $21.2(-6.4 \%)$ & $18.6(-17.8 \%)$ \\
c72 & ENS & 683 & $682(-0.2 \%)$ & $650(-4.9 \%)$ & $578(-15.4 \%)$ \\
\hline
\end{tabular}

In Bus 6, adding energy storage (DG+ESS) reduced ENS 2.8\%, while including load shedding (DG+LS) reduced this index $4.7 \%$. In contrast, the optimal coordinated operation of energy storage and 
load shedding (DG+ESS+LS) reduced ENS 12.7\%, this is 5.2\% more than the aggregated contribution of the other two cases $(12.7-2.8-4.7=5.2 \%)$. In the case of SAIDI, the additional improvement introduced by the optimal coordinated restoration is even more significant than for ENS: $9.6 \%(22.1-4-8.5=9.6 \%)$. With regard to Feeder c72 system, the additional improvement is $10.3 \%$ for ENS and $11.1 \%$ for SAIDI. Therefore, the reliability improvement introduced by the optimal coordinated operation of DGs, energy storage and load shedding was significantly larger than the improvements obtained by the individual operation of these resources, validating the methodology. In the following sections the results of the sensitivity analyses are presented to evaluate the contribution of the optimal coordination operating under different scenarios.

\subsection{Energy Storage Size Analysis}

The contribution of the optimal coordination on reliability is analysed for different sizes of energy storage (the case DG+ESS+LS is studied). Four values of capacity and other four of rated powers were evaluated and they were expressed by using their capacity and power ratios. These ratios represented the capacity and rated powers of the energy storage devices with regard to the nominal values in Table 1 (ratios of 1 correspond to values in Table 1).

Figure 5 shows the SAIDI and ENS indices obtained for the described analysis. In the figure, the largest evaluated energy storage size (ratios of 2) reduced SAIDI of Bus 6 system from 7.9 to $5.8 \mathrm{~h}$ /customer and ENS from 43.5 to $36.6 \mathrm{MWh} /$ year. For Feeder c72 system, SAIDI was reduced from 21.2 to $17.1 \mathrm{~h} /$ customer and ENS from 650 to $528 \mathrm{MWh}$ /year. These results proved that the size of energy storage has an important impact on the reliability improvement. However, this reliability improvement substantially depends on the combination of capacity and rated power as it can be seen in Figure 5. In addition, the reliability improvement tends to saturate at capacity and power ratios larger than 1.5. All these results highlight the importance of selecting adequate capacity and rated power of energy storage in order to meet a specific reliability improvement.

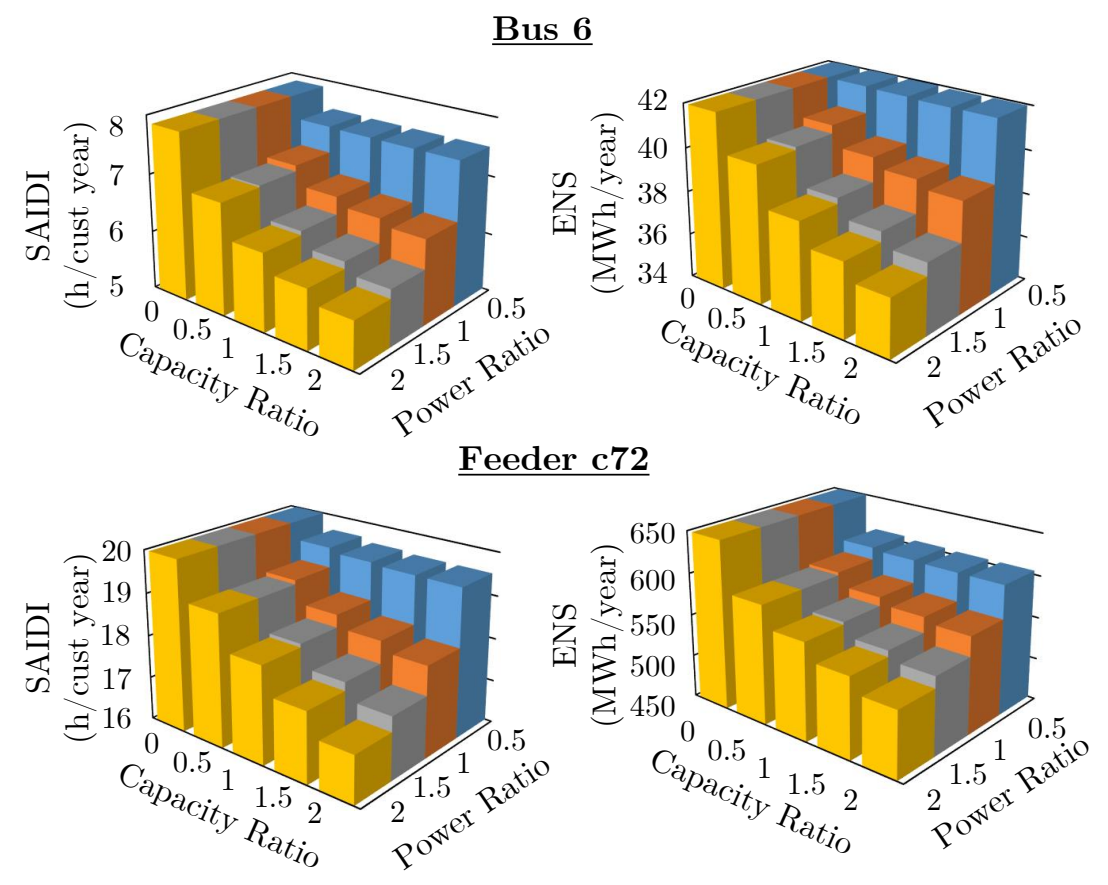

Figure 5. SAIDI and ENS of the test networks for different energy storage sizes.

\subsection{Load Shedding Analysis}

The impact of load shedding deployment on the test network reliability was also evaluated when the coordinated optimal restoration was performed. Three scenarios of load shedding deployment were 
analysed: Low, Medium and High. Table 3 shows the buses equipped with load shedding functionality (dispatchable loads) for each scenario, where Low scenario has the lower number of buses with load shedding and High scenario the largest number. In addition, each scenario was evaluated for the dispatchable loads equipped with 1,3 and 5 levels of load shedding $\left(\operatorname{set} S_{k, l}\right)$. The powers and priorities of the levels at a load point were assumed to be equal. Nominal size of energy storage (Table 1) was assumed for all the configurations.

Table 3. Buses with Dispatchable loads for the Scenarios of load shedding evaluation.

\begin{tabular}{lll}
\hline Scenario & Buses in Bus 6 & Buses in Feeder c72 \\
\hline Low & $16,26,30,32,34,35,37,38$ & $7-9,13,17-18,20,22-23$ \\
Medium & Low $+15,25,29,33,40$ & Low $+5,10,15,16,19$ \\
High & Medium $+17,27,28,31,36,39$ & Medium $+6,14,21,24$ \\
\hline
\end{tabular}

Figure 6 shows the SAIDI and ENS of the test networks for the load shedding analysis. Scenarios with Low, Medium and High load shedding deployment reduced ENS 11, 16 and 20\% in Bus 6 and 15, 25 and $43 \%$ for Feeder c72 (results given for 3 levels of load shedding and referred to the case without load shedding DG+ESS). SAIDI was also significantly reduced, although it was conditioned by the number of customers per load level. These results revealed the additional reliability improvement obtained by the increase of dispatchable loads in the networks and their optimal management.

With respect to the levels of load shedding per load point, increasing their number from 1 to 3 hardly improved reliability indices in Bus $6(1 \%)$. In the case of Feeder c72 system, this improvement presented relevant differences depending on the scenario: no improvement for Low, $3 \%$ for Medium and $25 \%$ for High. In contrast, in both test networks, increasing the number of levels from 3 to 5 had insignificant extra improvement. These differences were influenced by the power magnitude of the levels and demonstrate the need of considering this parameter in the reliability assessment.

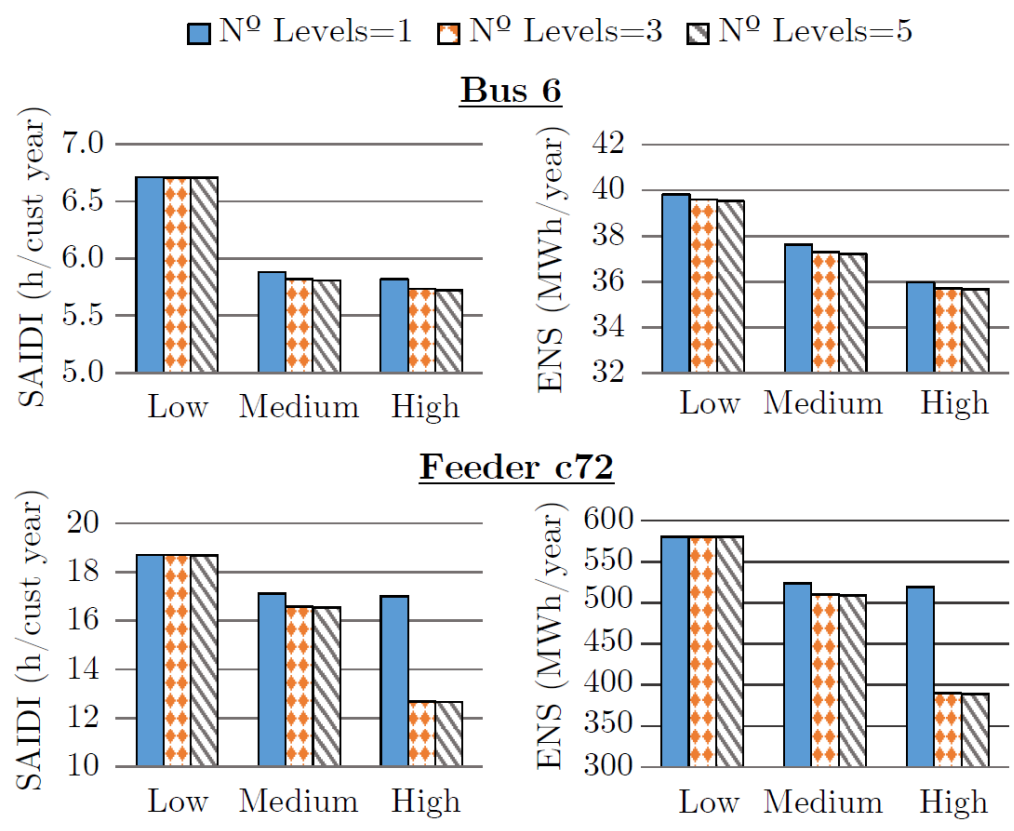

Figure 6. SAIDI and ENS for the scenarios of load shedding evaluation.

\subsection{Analysis of the Number of Interruptions}

In the previous analyses the maximum number of interruptions during a fault $(L I)$ was limited to one in order to avoid repetitive interruptions of customers. This section quantifies the impact of different $L I$ on the reliability indices. 
Three values of $L I$ were analysed: 1, 2 and 3. Results of the test networks for DG+ESS+LS case are shown in Table 4. Increasing LI from 1 to 2 in Bus 6 system (column \% LI 1-2 in Table 4) reduced (or improved) SAIDI $4.3 \%$ and ENS $2.3 \%$ but SAIFI increased (or worsened) $14 \%$. In the case of Feeder $\mathrm{c} 72$ system, the results were less favourable: SAIFI increased $22 \%$ but SAIDI and ENS were only reduced $2.6 \%$. Increasing LI from 2 to 3 minimally improved SAIDI and ENS but SAIFI worsened additional $6 \%$ in Bus 6 and 10\% in Feeder c72. Therefore, SAIDI and ENS can be improved by raising the $L I$ limit but the increase in SAIFI has to be taken into account as it can be more critical.

Table 4. Reliability indices for different numbers of permitted interruptions during a fault (LI).

\begin{tabular}{ccccccc}
\hline System & Index & $\boldsymbol{L I = 1}$ & $\boldsymbol{L I = 2}$ & $\boldsymbol{L I = 3}$ & $\mathbf{\%} \boldsymbol{L I ~ 1 - 2}$ & $\mathbf{\%} \boldsymbol{L I} \mathbf{1 - 3}$ \\
\hline \multirow{3}{*}{ Bus 6 } & SAIFI & 1.33 & 1.52 & 1.60 & $14 \%$ & $20 \%$ \\
& SAIDI & 6.71 & 6.42 & 6.42 & $-4.3 \%$ & $-4.4 \%$ \\
& ENS & 39.8 & 38.9 & 38.9 & $-2.3 \%$ & $-2.4 \%$ \\
\hline Feeder & SAIFI & 3.5 & 4.3 & 4.6 & $22 \%$ & $32 \%$ \\
c72 & SAIDI & 18.6 & 18.2 & 18.2 & $-2.6 \%$ & $-2.6 \%$ \\
& ENS & 578.2 & 563.2 & 562.9 & $-2.6 \%$ & $-2.6 \%$ \\
\hline
\end{tabular}

\section{Conclusions}

In this paper, a novel analytical methodology has been proposed to assess the reliability of active distribution networks with DGs, energy storage and dispatchable loads that are optimally coordinated under fault conditions. The optimal coordination of these three solutions during interruptions has been modelled and considered in reliability studies. For an accurate assessment, details of the optimal restoration strategy and the chronological fluctuations of renewable generation, demand and stored energy have been modelled and used in the evaluation. An analytical formulation has been proposed for the reliability assessment because of its superior computational efficiency compared to Monte Carlo simulation.

The effectiveness of the proposed methodology was validated by using two distribution networks. The results for both networks showed that DGs, energy storage and dispatchable loads can be used to enhance the network reliability. However, the optimal coordination of these resources additionally increased the reliability improvements between 5 and 10\% in the ENS (depending on the system) and approximately $10 \%$ for the SAIDI. The reliability results indicated that there is an important sensitivity with respect to the energy storage size and the number of installed dispatchable loads. In fact, increasing the number of dispatchable loads leads to ENS improvements between 10 and 43\%. In addition to that, the control level of dispatchable loads and the number of repetitive interruptions during a fault are also identified as factors affecting the reliability. The adequate selection of control levels of dispatchable loads, in particular, shows important effect in the reliability. Increasing the number of levels from 1 to 3 results in ENS improvements up to $25 \%$. Therefore, all these parameters should be properly considered when the optimal coordinated restoration is applied. Moreover, the proposed detailed optimal restoration shows huge application potential for future studies and implementations. The main topics for future research include the integration of other restoration strategies in reliability studies and the cost-benefit evaluation of the technologies analysed in this paper.

Author Contributions: Alberto Escalera designed and conceived the paper, developed and implemented the methodology, obtained the results and analyzed the data. Edgardo D. Castronuovo and Milan Prodanovic supervised the research and the obtained results. All the authors defined the structure of the paper. Alberto Escalera and Javier Roldan-Perez wrote the paper. Edgardo D. Castronuovo, Milan Prodanovic and Javier Roldan-Perez revised the paper.

Funding: This research was funded by the research program "Energy and Environment 2018" of Fundación Iberdrola, project name "SinCortes".

Conflicts of Interest: The authors declare no conflict of interest. 


\section{References}

1. Allan, R.; Billinton, R. Probabilistic assessment of power systems. Proc. IEEE 2000, 88, 140-162. [CrossRef]

2. Billinton, R.; Ali, S.; Wacker, G. Rural distribution system reliability worth evaluation using individual customer outage cost characteristics. Int. J. Electr. Power Energy Syst. 2004, 26, 235-240. [CrossRef]

3. Shang, Y. Resilient Multiscale Coordination Control against Adversarial Nodes. Energies 2018, 11, 1844. [CrossRef]

4. Shang, Y. Resilient consensus of switched multi-agent systems. Syst. Control. Lett. 2018, 122, 12-18. [CrossRef]

5. The Need for a Fundamental Review of Electricity Networks Reliability Standards; ETP SG Security And Resilience Task Force, 2016. Available online: https://www.etip-snet.eu/wp-content/uploads/2017/04/ETP_SG_ Future_Network_Reliability_Standards_2016.pdf (accessed on 19 August 2019).

6. Nigim, K.A.; Hegazy, Y.G. Intention islanding of distributed generation for reliability enhancement. In Proceedings of the 2003 IEEE Power Engineering Society General Meeting (IEEE Cat. No. 03CH37491), Toronto, ON, Canada, 13-17 July 2003; Volume 4, p. 2451.

7. Arya, R.; Choube, S.; Arya, L. Reliability evaluation and enhancement of distribution systems in the presence of distributed generation based on standby mode. Int. J. Electr. Power Energy Syst. 2012, 43, 607-616. [CrossRef]

8. Escalera, A.; Hayes, B.; Prodanović, M. A survey of reliability assessment techniques for modern distribution networks. Renew. Sustain. Energy Rev. 2018, 91, 344-357. [CrossRef]

9. Zhou, Y.; Mancarella, P.; Mutale, J. Modelling and assessment of the contribution of demand response and electrical energy storage to adequacy of supply. Sust. Energy Grids Netw. 2015, 3, 12-23. [CrossRef]

10. Billinton, R.; Allan, R. Reliability Evaluation of Power Systems, 2nd ed.; Plenum: New York, NY, USA, 1996.

11. Atwa, Y.M.; El-Saadany, E.F. Reliability Evaluation for Distribution System With Renewable Distributed Generation During Islanded Mode of Operation. IEEE Trans. Power Syst. 2009, 24, 572-581. [CrossRef]

12. Bie, Z.; Zhang, P.; Li, G.; Hua, B.; Meehan, M.; Wang, X. Reliability Evaluation of Active Distribution Systems Including Microgrids. IEEE Trans. Power Syst. 2012, 27, 2342-2350. [CrossRef]

13. Al-Muhaini, M.; Heydt, G.T. Evaluating Future Power Distribution System Reliability Including Distributed Generation. IEEE Trans. Power Deliv. 2013, 28, 2264-2272. [CrossRef]

14. Zou, K.; Agalgaonkar, A.P.; Muttaqi, K.M.; Perera, S. An Analytical Approach for Reliability Evaluation of Distribution Systems Containing Dispatchable and Nondispatchable Renewable DG Units. IEEE Trans. Smart Grid 2014, 5, 2657-2665. [CrossRef]

15. Escalera, A.; Prodanović, M.; Castronuovo, E.D.; Mazuera, J.C. A Comparison of The Renewable Distributed Generation Models used in Reliability Assessment. In Proceedings of the 2018 IEEE International Conference on Probabilistic Methods Applied to Power Systems (PMAPS), Boise, ID, USA, 24-28 June 2018; pp. 1-6.

16. Conti, S.; Nicolosi, R.; Rizzo, S.A. Generalized Systematic Approach to Assess Distribution System Reliability with Renewable Distributed Generators and Microgrids. IEEE Trans. Power Deliv. 2012, 27, 261-270. [CrossRef]

17. Wang, S.; Li, Z.; Wu, L.; Shahidehpour, M.; Li, Z. New Metrics for Assessing the Reliability and Economics of Microgrids in Distribution System. IEEE Trans. Power Syst. 2013, 28, 2852-2861. [CrossRef]

18. Chen, C.; Wu, W.; Zhang, B.; Singh, C. An Analytical Adequacy Evaluation Method for Distribution Networks Considering Protection Strategies and Distributed Generators. IEEE Trans. Power Deliv. 2015, 30, 1392-1400. [CrossRef]

19. Xu, Y.; Singh, C. Adequacy and Economy Analysis of Distribution Systems Integrated With Electric Energy Storage and Renewable Energy Resources. IEEE Trans. Power Syst. 2012, 27, 2332-2341. [CrossRef]

20. Narimani, A.; Nourbakhsh, G.; Ledwich, G.; Walker, G. Optimum electricity purchase scheduling for aggregator storage in a reliability framework for rural distribution networks. Int. J. Electr. Power Energy Syst. 2018, 94, 363-373. [CrossRef]

21. Escalera, A.; Prodanović, M.; Castronuovo, E.D. Analytical methodology for reliability assessment of distribution networks with energy storage in islanded and emergency-tie restoration modes. Int. J. Electr. Power Energy Syst. 2019, 107, 735-744. [CrossRef]

22. Farzin, H.; Fotuhi-Firuzabad, M.; Moeini-Aghtaie, M. Stochastic Energy Management of Microgrids During Unscheduled Islanding Period. IEEE Trans. Ind. Inform. 2017, 13, 1079-1087. [CrossRef] 
23. Moeini-Aghtaie, M.; Farzin, H.; Fotuhi-Firuzabad, M.; Amrollahi, R. Generalized Analytical Approach to Assess Reliability of Renewable-Based Energy Hubs. IEEE Trans. Power Syst. 2017, 32, 368-377. [CrossRef]

24. Wang, P.; Li, W. Reliability evaluation of distribution systems considering optimal restoration sequence and variable restoration times. IET Gener. Transm. Distrib. 2007, 1, 688-695. [CrossRef]

25. Billinton, R.; Wang, P. Teaching distribution system reliability evaluation using Monte Carlo simulation. IEEE Trans. Power Syst. 1999, 14, 397-403. [CrossRef]

26. Safdarian, A.; Degefa, M.Z.; Lehtonen, M.; Fotuhi-Firuzabad, M. Distribution network reliability improvements in presence of demand response. IET Gener. Transm. Distrib. 2014, 8, 2027-2035. [CrossRef]

27. Farzin, H.; Fotuhi-Firuzabad, M.; Moeini-Aghtaie, M. Role of Outage Management Strategy in Reliability Performance of Multi-Microgrid Distribution Systems. IEEE Trans. Power Syst. 2018, 33, 2359-2369. [CrossRef]

28. Hamzah, B.N. Islanded Operation of Distribution Networks; Technical Report; Distributed Generation Coordinating Group (DGCG), 2005. Available online: www.distributedgeneration.gov.uk (accessed on 16 August 2019).

29. Allan, R.N.; Billinton, R.; Sjarief, I.; Goel, L.; So, K.S. A reliability test system for educational purposes-basic distribution system data and results. IEEE Trans. Power Syst. 1991, 6, 813-820. [CrossRef]

30. Ćurčić, S.; Ozveren, C.S.; Crowe, L.; Lo, P. Electric power distribution network restoration: A survey of papers and a review of the restoration problem. Electr. Power Syst. Res. 1995, 35, 73-86. [CrossRef]

31. Chowdhury, A.; Koval, D. Power Distribution System Reliability: Practical Methods and Applications; John Wiley \& Sons, Inc.: Hoboken, NJ, USA, 2009.

32. IEEE Guide for Electric Power Distribution Reliability Indices; IEEE Std 1366, 2001 ed.; IEEE: Piscataway, NJ, USA, 2001.

33. Macedo, L.H.; Franco, J.F.; Rider, M.J.; Romero, R. Optimal Operation of Distribution Networks Considering Energy Storage Devices. IEEE Trans. Smart Grid 2015, 6, 2825-2836. [CrossRef]

34. Billinton, R.; Jonnavithula, S. A test system for teaching overall power system reliability assessment. IEEE Trans. Power Syst. 1996, 11, 1670-1676. [CrossRef]

35. European Commision SmartHG Project Website. Available online: http://smarthg.di.uniroma1.it/ (accessed on 16 August 2019). 